\title{
LA QUIEBRA DE LAS UNANIMIDADES TEORICAS: LAS RELACIONES DE ROLES
}

\author{
Rafael Gobernado
}

Universidad de Málaga

RESUMEN: Se esboza, aquí, una hipótesis basada en un concepto poco desarrollado en la práctica sociológica, el de relaciones de roles. Por tal concepto se entiende la definición del rol que efectúa el propio actor, eligiendo entre dos alternativas que tienden a ser contradictorias. La hipótesis en cuestión puede ayudar a explicar el origen del rompimiento de categorías sociales aparentemente homogéneas.

El objetivo del texto que sigue no es otra cosa que intentar sacar del olvido una noción ciertamente interesante como es la de relaciones de roles, expresión que aquí se utiliza aunque también se conozca por otras semejantes, tales como la de relaciones de rol o la de rol relacional. Se ha de advertir, no obstante, que tal terminología puede ser más causa de confusión que de aclaración, ya que, en principio, todo rol puede ser definido en términos de relación ${ }^{1}$, con lo que parece a primera vista que se está cometiendo una redundancia, cuando verdaderamente nos estamos refiriendo a un tema específico dentro del análisis de roles.

1 No es ésta la única forma de definir los roles. En líneas generales, como se sabe, hay dos extremos entre los que pueden situarse las diferentes conceptualizaciones del término en cuestión: por un lado, la estructural, de acuerdo con la cual los roles son realidades externas a los sujetos a manera de celdas en los que se desenvuelven; y la relacional, que defiende la idea de que los roles se crean y recrean constantemente en el proceso de interacción cotidiana. La realidad, seguramente, participa de ambos puntos de vista. 
Se trata de un concepto, el de las relaciones de roles, al que los autores han dedicado muy poca atención ${ }^{2}$ y que, como resultado de ello, quizá, no ha sido definido con la precisión que merece. Incluso debería hablarse más bien de hipótesis que de concepto, dada la complejidad de relaciones que encierra y la escasa o nula verificación empírica de las mismas.

La hipótesis en cuestión podría materializarse en el siguiente ejemplo deducido del texto de T. Parsons que luego se cita. Moviéndonos en el campo de lo especulativo, y de acuerdo con la idea que nos traemos entre manos, hemos de presumir que si sacamos de varias aulas a todos aquellos muchachos que hayan sido definidos como «buenos alumnos» y formamos otra aula con todos ellos, el resultado a la larga no sería una clase escolar homogénea formada por «buenos alumnos», sino que volvería a reproducirse la división inicial entre «buenos» y «malos alumnos». Lo propio ocurriría si empezáramos de nuevo con una clase homogénea formada por «malos alumnos»; con el tiempo aparecería la consabida distinción entre «buenos» y «malos». El problema se complica cuando se advierte que no siempre son los mismos alumnos, dentro de igual aula, los que merecen el calificativo de «buenos» o «malos», sino que un mismo alumno puede cambiar sucesivamente de una categoría a otra. Todo ello con independencia de que varíe o no la proporción general entre el número de unos y de otros.

El punto de partida para la comprensión de tal fenómeno sería la consideración del proceso como resultado de condiciones sociales, no de rasgos de la personalidad, aunque, como es claro, éstos jueguen cierto papel de importancia, papel que no nos incumbe a nosotros destacar.

$\mathrm{Y}$ aquí es donde tiene sentido sacar a colación el concepto de relaciones de roles. La noción en cuestión tiene que ver con el proceso de aprendizaje o de interiorización, así como con el de definición de roles. Por lo tanto, ha de ser considerado como un aspecto más, aunque de singular importancia, como veremos, dentro del complejo tratamiento de tales temas. Como se sabe, los roles o papeles sociales ${ }^{3}$ se presentan en conjuntos formando lo que los estudiosos del tema llaman campos de roles. Tales campos están constituidos por roles recíprocos, complementarios, dando lugar a unida-

${ }^{2}$ Como ejemplo de esa falta de atención sobre el tema destaca el libro de R. Boudon, La lógica de lo social, Ed. Rialp, Madrid, 1981, pp. 66-92. En el capítulo III de tal libro, el autor repasa aquellos aspectos de la noción del rol que posibilitan la libertad del actor que lo representa: llama la atención sobre la complejidad, la variabilidad, la ambivalencia, así como sobre otros problemas propios de la definición de cualquier rol. No obstante, no se refiere, en ningún momento, al problema de las relaciones de rol que aquí se trata, el cual hubiera encajado perfectamente en su discurso.

${ }^{3}$ El libro clave para la comprensión del concepto de rol es el de R. DaHrendorf, Homo sociologicus, Madrid, Akal, 1975. Se trata de un concepto demasiado conocido por la Sociología como para entrar en su conceptualización. No obstante, los manuales de Sociología, que tantas páginas suelen dedicar a la definición del mismo, ignoran totalmente el tema que nos ocupa, el de las relaciones de roles. 
des sociales de rango más general que la del propio rol4. Precisamente, debido a ello, los roles no se interiorizan de forma aislada, sino que, más bien, se interiorizan relaciones de roles ${ }^{5} \mathrm{o}$, lo que es igual, conjuntos de roles relacionados. Asimismo, en cada sistema social pueden establecerse tantas relaciones cuantos pares de roles existan en el mismo.

De acuerdo con ello, cada papel puede ser definido desde dos puntos de vista que tienden a ser contradictorios, de acuerdo con cada uno de los pares de roles existentes en el sistema social acotado: el punto de vista del rol propio y el del contrario. Más concretamente y en último extremo: el punto de vista de los «iguales» ${ }^{6}$ y el del rol recíproco.

\section{LA IDENTIFICACION CON LOS «LOS IGUALES» O CON «EL OTRO»}

La particularidad del asunto radica en que en el proceso de interiorización, proceso que consiste en asumir el papel, el actor social termina por identificarse con una de las partes de la relación (o de la pareja de roles relacionados), no necesaria ni lo más probablemente la «suya». En otras palabras, el actor social puede identificarse, o bien con sus «iguales», o bien con el otro elemento de la relación, ya que, en la práctica, como se dijo, las opciones suelen expresarse en opciones duales. El proceso de identificación se repite, en teoría, para cada par de roles del campo correspondiente.

4 Cuando esos conjuntos de roles recíprocos satisfacen necesidades fundamentales, forman las llamadas instituciones.

5 Esto es, el hijo no aprende sólo su papel social, sino que aprende también el de «padre»; el alumno aprende tanto el papel social de «alumno» como el de «maestro»; el marido aprende tanto su propio papel como el de «esposa», etc. Esto es obvio. Volviendo a la metáfora teatral en la que se basa el concepto de rol, cada actor debe conocer tanto su propio papel como el papel del actor al que da respuesta. Pero no sólo interioriza ambos papeles, sino que también aprende lo que espera cada papel del otro: el hijo aprende lo que el padre espera del hijo; el alumno, lo que el maestro espera del alumno; el marido, lo que la esposa espera del marido, etc.

${ }^{6}$ El concepto de grupo de «iguales» ha sido abundamentemente utilizado en las ciencias sociales, en particular por la antropología. Con êl se quiere dar a entender a una agrupación de carácter comunitario, con un profundo sentimiento del «nosotros», organizada en torno a algún rasgo común de sus miembros, y con valores y preferencias comunes. Ejemplos del mismo son el grupo de «amigotes»; los grupos de edad en las aldeas; los compañeros de trabajo, etc. Este mismo sentido es el que recibe generalmente en este texto, ya que la definición del rol de acuerdo con los «iguales» termina por servir de apoyo para la formación del propio grupo de «iguales». No obstante, a veces se deja entrever la cualidad de «cuasigrupo» en el término aludido y, por lo tanto, la identificación con tal cuasigrupo tampoco puede ser tan cerrada como en el caso anterior. Por razones poco claras, los hombres están más inclinados que las mujeres a organizarse en tales grupos.

7 Así, el alumno puede identificarse, bien con sus «iguales» (compañeros de clase), o bien con el profesor; el hijo puede hacer lo propio, bien con sus «iguales» (hermanos), o bien con el padre, etc. 
El resultado más evidente de la identificación con los «iguales» es la progresiva formación de un grupo de tales (en último extremo, organizado y con clara conciencia del nosotros), cuyos miembros, generalmente, son contrarios a los que han adoptado una identificación con el rol recíproco. Por su parte, la organización de estos últimos será una consecuencia de la organización de los «iguales», es decir, su organización irá a remolque de la primera. De cualquier forma, entre ambos colectivos se establecerá una típica relación dialéctica.

La identificación supone, en principio, una preferencia de carácter no racional ni reflexivo. Supone, asimismo, tomar como modelo a seguir el punto de vista del extremo adoptado. No se trata de obrar «como si» se representara uno u otro papel, sino de definir el comportamiento esperado del papel en cuestión desde el punto de vista de la opción elegida. En el proceso de identificación, el actor social elige el punto de vista de uno u otro elemento de la relación dual de roles para definir el contenido del rol que ha de representar.

En principio, la adopción del punto de vista de los «iguales» a la hora de definir el propio rol implica una fuente de tensión constante; mientras que la adopción del punto de vista del rol recíproco favorece la estabilidad y el orden dentro del mismo. Evidentemente, si nos comportamos como desea la persona con la que convivimos, seguramente tendremos menos motivos de fricción con ella que si no lo hacemos así. Pero la tensión se establece también entre las dos categorías sociales que surgen a raíz de la doble identificación dentro del mismo rol: entre los que se identifican con los «iguales», por un lado, y los que se identifican con el rol recíproco, por otro.

El problema se radicaliza en la medida en que la definición del comportamiento esperado por cualquiera de las dos opciones tiende a convertirse en la contraria del otro. Es decir, la configuración de cómo debe comportarse un individuo por desempeñar un papel social termina por ofrecer dos alternativas contradictorias, opuestas. Una de ellas tenderá a facilitar las relaciones entre los dos roles, mientras que la otra traerá fácilmente el conflicto entre los mismos. Tal proceso de radicalización (de ideas) parece consustancial al proceso de bipolarización (de grupos) de las opciones tratadas. Todo ello supone que, cualquiera que sea el sistema social observado, siempre cabe la posibilidad de ruptura interna a partir de la doble identificación anteriormente apuntada.

Tal proceso generador de tensión arriba esbozado es el que otorga singular importancia a esta doble definición del rol, oscureciendo y quitando interés a las demás diferencias de definición de un mismo rol que puedan apreciarse en cualquier organización social. Pero, antes de matizar más, conviene atender al uso que dos autores de singular prestigio han dado al concepto tratado: T. Parsons y E. Bott. 


\section{T. PARSONS: EL ROL DEL ALUMNO}

En 1959 T. Parsons publicó un artículo que hace referencia directa al tema de las relaciones de roles: «La clase como sistema social» ${ }^{8}$, en el que «clase» es sinónimo de «aula escolar». En el citado artículo, Parsons establece un conjunto de relaciones significativas en el proceso de socialización infantil entre tres conjuntos de elementos: la familia, la escuela y el grupo de iguales. Con motivo de ello, Parsons se adentra en la definición del concepto de relaciones de rol, definición que no termina de clarificar el concepto citado. Permítaseme que cite largamente al autor:

«... el aprendizaje de la motivación del logro es, psicológicamente hablando, un proceso de identificación con el profesor que impulsa a obrar bien en clase con el fin de agradar a la profesora - a menudo apoyada por los padres - en el mismo sentido en que el niño, en la edad preedípica, aprende nuevas habilidades con el fin de agradar a su madre.

Respecto a esto, sostengo que lo que se ha interiorizado, por medio del proceso de identificación, es un modelo recíproco de relaciones de rol. A menos que la interiorización fracase totalmente, no solamente se interiorizará un aspecto de la acción, sino los dos. Se pondrá, sin embargo, el acento en uno u otro, de suerte que ciertos niños se identificarán más con el agente socializador, y otros con el rol opuesto. Así, en la edad preedípica, el niño independiente se identifica más con su padre o su madre, mientras que el niño "dependiente" se identifica con el rol de niño en relación con uno u otro de sus padres.

En la escuela, el profesor se halla definido institucionalmente como superior a todo alumno en el plano del conocimiento de las materias del programa, así como en el de su responsabilidad como "buen" ciudadano de la escuela.

En la medida en que la clase tiende a dividirse en dos - aunque esta dicotomía está lejos, desde luego, de ser absoluta-, tiende a hacerlo en general, por una parte, sobre la base de una identificación con el profesor o sobre el reconocimiento de su rol como modelo, y por otra, sobre la identificación con el grupo de iguales del alumno.

8 T. PARSONS, «La clase como sistema social: algunas de sus funciones en la sociedad americana», en A. GRAS, Textos fundamentales de Sociología de la educación, Madrid, Narcea, 1976, pp. 53-60. La primera edición de este artículo tuvo lugar en la Harvard Educational Review, 29, 4, 1959. Llama la atención el hecho de que se haya ignorado esta fuente de conflicto en el planteamiento teórico parsoniano, tan escaso, en general, de tal tipo de planteamientos. Véase, por ejemplo, J. Almaraz, «Conflicto y teoría sistemática: EI conflicto del último Parsonss, en J. Pino Artacho (coord.), El conflicto social, Málaga, Universidad de Málaga, 1988. 
Esta división de la clase, en función de una identificación con el profesor o con el grupo de iguales, corresponde de una manera tan chocante a la distribución de los alumnos destinados al colegio [a pasar al nivel superior] y los que no lo son, que no puede impedirse emitir la hipótesis de que esta dicotomía estructural a nivel de sistema escolar es la primera fuente de la dicotomía selectiva...» (p. 55).

En líneas generales, la idea de Parsons, limitada al aula escolar, es que ésta se divide en alumnos «buenos» y alumnos «malos» de forma espontánea y de acuerdo con el proceso de identificación con el profesor o con los iguales, respectivamente. La identificación, recordemos, es un proceso no racional ni consciente, independiente de las aptitudes e inteligencia del muchacho. Los alumnos que se identifican con el profesor tienden a trabajar; a estudiar; a cumplir con lo que el profesor espera del alumno. Por el contrario, el muchacho que se identifica con sus iguales tiende a comportarse «mal» frente al profesor; a rechazar la disciplina que éste pretende imponer, etc. El resultado es que el éxito o el fracaso escolar encuentran un primer e importante punto de apoyo en tal proceso de identificación. Se trata, de acuerdo con el autor, de un proceso espontáneo e inevitable.

La explicación que encuentra Parsons a tal diferenciación interna escolar es la presencia o ausencia de un rasgo de carácter en la personalidad de los alumnos: el ser o no independiente. U. Bronfenbrenner, ocho años después, llegó al convencimiento de que es el sistema de valores, la cultura en su sentido antropológico más amplio, lo que explica la tendencia a identificarse preferentemente con el profesor o con sus iguales. Así, después de comparar niños de EE.UU. y de la Unión Soviética, llegó a la conclusión, entre otras, que los niños occidentales tendían más a identificarse con sus iguales; mientras que los niños soviéticos lo hacían con sus maestros?.

\section{E. BOTT: EL ROL CONYUGAL}

La primera edición del libro de E. Bott que aquí nos concierne, el de Familia y red social, tuvo lugar en Londres en 1957. Desde entonces se ha convertido en un clásico de la bibliografía sobre redes sociales. Pese a que algunas críticas al libro se dirigieron precisamente a poner de manifiesto

${ }^{9} \mathrm{U}$. BRONFENBRENNER, «Respuesta ante la presión de iguales de edad versus adultos en los niños soviéticos y americanos», en J. R. Torregrosa y J. R. Crespo, Estudios básicos de psicología social, Madrid, Hora y CIS, pp. 479-492. 
que la definición que daba de rol relacional era un tanto vaga e imprecisa, vale la pena que la recordemos:

«Un rol relacional - dice Bott- está formado por aquellos aspectos de una relación que suponen expectativas recíprocas de comportamiento de una persona respecto a otra. Mediante esta definición se trata de excluir una gran variedad de expectativas idiosincrásicas y de significados personales que acompañan a las relaciones mantenidas por los individuos. Pero en el caso de las relaciones familiares, la línea que separa las expectativas institucionalizadas formales y las expectativas individuales es, con frecuencia, difícil de trazar... De hecho, el término rol relacional se refiere a aquellas expectativas recíprocas de conducta que son consideradas por el marido y la mujer como propias de su círculo social» $(\mathrm{p}, 37)$.

Y más adelante establece la siguiente clasificación de roles relacionales conyugales:

«Utilizamos la expresión rol relacional conyugal separado para dar a entender una relación en la que predominan los tipos complementario e independiente de organización. Marido y mujer mantienen una diferenciación clara de tareas y una gran cantidad de aficiones y de actividades separadas; tienen claramente definida la división del trabajo en tareas femeninas y tareas masculinas; cuentan con tener maneras diferentes de pasar el tiempo, y el marido tiene sus propios amigos fuera de casa, y la mujer, sus amigas. Con la expresión rol relacional conyugal conjunto se quiere dar a entender una relación en la que predomina, relativamente, la organización conjunta. El marido y la mujer confían en realizar la mayor parte de las actividades juntos con un mínimo de diferenciación en las tareas y de separación en las aficiones. No solamente planifican los asuntos familiares conjuntamente, sino que se alternan en muchas tareas domésticas y pasan mucho tiempo libre juntos» (pp. 92 y 93).

En la definición anterior se sacan en claro algunas cuestiones. En primer lugar, que se trata de un problema propio del sistema social y no de la personalidad de los autores. Además que se trata de comportamientos orientados hacia otra persona que ocupa un rol complementario en el campo de roles acotado, en este caso la familia. También, aunque con otras palabras, tal orientación puede guiarse bien desde el punto de vista de los iguales (los hombres por un lado y las mujeres por otro), bien desde el punto de vista del otro complementario. No queda claro, sin embargo, si se trata de dos definiciones de un mismo rol (tal y como lo definimos nosotros) o de dos roles diferentes (versión que parece coincidir con la última 
parte de la cita). Tampoco queda claro si se trata de un rol común a dos personas (ambos cónyuges) o es individual.

De cualquier forma, se aprecia que consiste en una elección de expectativas de comportamiento, elección, en cierta medida, obligada por el sistema social. Así, y de acuerdo con la autora, si se pertenece a una red poco unida (en la que los miembros apenas tienen relaciones entre sí), el rol tenderá a ser conjunto; por el contrario, si se pertenece a una red muy unida (en la que los miembros establecen muchas relaciones mutuas), el rol tenderá a ser separado. A su vez, la pertenencia a un tipo u otro de red viene dado por la frecuencia de movilidad espacial no recurrente que haya sufrido la familia considerada ${ }^{10}$. En este caso, la autora propone un modelo explicativo de la elección efectuada más convincente que el de T. Parsons.

\section{CONCEPTUALIZACION DE LAS RELACIONES DE ROLES}

A partir de los antecedentes citados, es posible establecer una conceptualización más precisa del término en cuestión. En principio, hay que repetir que se trata de diversas opciones de definición de un mismo rol, por parte del actor social que ha de representarlo. Cada opción, a su vez, se corresponde con el punto de vista que se presume para cada uno de los roles, organizados en parejas, que conforman el campo de roles en el que se mueve: el «propio» y el «contrario». La aceptación del primero dará lugar a la identificación con los «iguales», en su caso más extremo.

Es de presumir, por lo tanto, que tal fenómeno podrá encontrarse en todos los pares de roles posibles dentro del sistema, aunque no todos tendrán el mismo interés en el análisis de éste. De la misma forma, afectará también a los dos roles de cada pareja, cada uno de los cuales sufrirá el proceso de definición doble, aunque seguramente tenga más interés uno que otro en el análisis citado.

No obstante la previsión teórica anterior, es obvio que las diferencias entre una y otra definición del mismo rol dependen de las relaciones que se espera que establezcan tales roles entre sí. En la medida en que no se entrecruzan los comportamientos esperados, no hay motivo para intentar adecuar la conducta esperada del uno al otro. Por el contrario, en la

${ }^{10}$ No es éste el momento de abordar la conocida hipótesis de E. Bott sobre las redes sociales y su influencia en el comportamiento esperado de los individuos. Véase E. BotT, Familia y red social, Madrid, Taurus, 1990. 
medida en que se esperan comportamientos recíprocos, cada rol ${ }^{11}$ preferirá adecuar el otro a sí mismo, antes que al contrario.

Aquí radica la primera fuente de tensión entre las posibles definiciones del mismo rol, ya que, en principio, las definiciones que aquí se tratan no parecen compatibles. Una vez iniciada la oposición entre ellas, es fácil prever, bajo ciertas condiciones, una progresiva tendencia hacia la polarización, la radicalización y la homogeneización interna de cada una de tales definiciones. Así, volviendo al aula escolar de T. Parsons, se puede presumir que las definiciones del comportamiento esperado del «alumno bueno» (el que se identifica con el profesor o, en otras palabras, que define el rol de alumno de acuerdo con el punto de vista del profesor) y el del «alumno malo» (aquel que define el rol de acuerdo con sus «iguales»), una vez iniciadas, tenderán a volverse cada vez más nítidamente diferenciadas, es decir, tenderán a tener cada vez menos elementos comunes entre sí; al mismo tiempo que irán desapareciendo las dudas sobre qué comportamientos son los esperados para cada una de ellas; todo ello favorecerá, de paso, la bipolarización de las opciones, ya que el conflicto es cosa fundamentalmente de dos, no de tres o más elementos ${ }^{12}$. El resultado final es la aparición de una definición propia del grupo de «iguales», frente a otra definición propia del rol recíproco.

¿Hasta qué punto puede generalizarse más allá del aula esta sospecha? De acuerdo con nuestra hipótesis, cualquier conjunto homogéneo, cualquier categoría social delimitada en función de la realización de un mismo rol, tenderá a la división de sus efectivos de acuerdo con las definiciones, inevitablemente encontradas, del citado rol: la de los «iguales» y la del rol recíproco. En resumen, no es posible la unanimidad. De ahí que aparezcan como utopías abundantes conceptos utilizados por la sociología, los cuales dan por sentado la existencia de colectividades internamente homogéneas por definición, ya sean estratos, clases o categorías sociales definidas en razón del sexo, la edad u otras.

Ahora bien, el problema es tanto práctico como teórico. En efecto, en el caso de ser válida la hipótesis apuntada, no cabría la posibilidad de desarrollar organizaciones sociales, del tamaño que fueran, en la que todos los miembros se encontraran de acuerdo con los elementos superiores en la jerarquí; en otras palabras, no habría posibilidad de que un sistema estuviera legitimado completamente. Siempre babria que esperar la contesta-

1 Ha de quedar claro que los roles «no prefieren nada», sólo prefieren las personas que los representan. Ahora bien, en la medida en que el rol, o comportamiento esperado, es consecuencia inmediata de la continua y constante realización del rol por parte de los actores sociales de un sistema, el rol se verá modificado en la dirección que marquen habitualmente tales actores.

${ }_{12}$ Como es obvio, el tema en cuestión encaja fácilmente dentro de la teoría general del conflicto. Véase J. DEL Pino ARTACHO, «El conflicto social en la teoría sociológica», en J. DEL Pino Artacho (coord.), El conflicto social, ob. cit., pp. 161-200. 
ción, y por un motivo, en general, no tenido en cuenta por los que habitualmente atienden a la contestación como algo normal. No se trataría únicamente de conflicto de intereses (la lucha de clases, por ejemplo) ni de estructuras emergentes producto de tomas de decisión racional (recuérdese al individualismo metodológico), sino de motivaciones espontáneas no racionales. Habría que cuestionarse, incluso, la propia contestación, ya que en la medida en que ha de organizarse para tener éxito, daría pie a las condiciones para su propia contestación interna.

Tal punto formal de partida nos lleva a plantearnos dos preguntas básicas, más bien una sola, pero de dos maneras diferentes: ¿por qué unos escogen una opción (se identifican con un rol) y otros escogen la otra (se identifican con el otro rol)? Y la otra: ¿por qué varían las proporciones de los que escogen una u otra opción? Ya se indicó que el proceso de identificación no es consciente ni racional y, seguramente, los rasgos de la personalidad tengan singular importancia en el mismo. Sin embargo, nuestra hipótesis es que se trata de un proceso guiado, dentro de ciertos límites, por las necesidades del sistema social en orden a su adaptación o supervivencia, en último extremo. Al igual que la variación en las proporciones de los que eligen una u otra opción de las que se ofrecen. Se trata, en términos generales, de un proceso espontáneo de diferenciación interna de las categorías sociales, inicialmente homogéneas, para obtener constantemente una alternativa posible a la situación imperante. Ha de ser considerado como un simple mecanismo de defensa ante los posibles cambios de la estructura social, para poder disponer, de inmediato, de individuos capaces de responder correctamente a una nueva situación social opuesta a la anterior. En resumen, es una consecuencia de la labilidad de las estructuras sociales, así como de la necesidad de continuación que tienen las mismas.

El tamaño de la proporción de individuos que adopten una definición del rol diferente a la «habitual» hasta un momento dado, así como la radicalización del enfrentamiento entre ambas definiciones, dependerá de múltiples factores:

a) Primeramente tiene que ver con la situación de estabilidad o crisis en la que se encuentre el segmento de la estructura social correspondiente. De cualquier forma, por mucha estabilidad de la que goce tal segmento, en ningún momento dejará de existir una opción «contraria», por apagada que esté.

b) Además, es de presumir que en situaciones de pertenencia voluntaria a categorías sociales, las definiciones de un mismo rol serán menos distantes y contrarias que las definiciones efectuadas en situaciones de pertenencia obligatoria. Así, por ejemplo, dentro del mismo ejército, la polarización y radicalización de las definiciones será mucho mayor entre los soldados de leva forzosa que entre los soldados voluntarios profesionales. 
c) De la misma forma, en la medida en que los actores dispongan de una mayor capacidad de toma de conciencia de la realidad, también disminuirá la radicalización entre las definiciones consabidas. Este es el caso, por ejemplo, de los alumnos de los cursos superiores en la educación obligatoria, frente a los alumnos de los primeros cursos, de menor edad y, por lo tanto, más susceptibles de adopción de posturas contradictorias en la definición de roles.

d) Asimismo, habrá más posibilidades de polarización cuando los roles correspondientes forman parte de una relación acusadamente jerarquizada que cuando no existe tal jerarquía.

e) Por último, es de presumir que los conflictos sociales ajenos al que nos ocupa también pueden afectar a la diferenciación de opciones de definiciones de un mismo rol.

Nos queda por responder la primera pregunta. Ya suponemos que el tema que nos ocupa es un mecanismo de adaptación y defensa frente a posibles cambios en la estructura social o en parte de ella, pero ¿cómo se consigue? ¿Por qué un individuo escoge de forma tan poco racional una opción en aras de una supuesta utilidad para el sistema social? Hemos de pensar que se trata de un proceso que tiene lugar a nivel individual como consecuencia de la interacción cotidiana, y que tiene que ver con la «definición de la situación» que realice, de forma intuitiva y vaga, el propio actor a partir de tal interacción cotidiana.

\section{LA QUIEBRA DE TOTALIDADES TEORICAS: UN EJEMPLO}

Como se afirmó antes, son muchas las ocasiones en las que los sociólogos utilizan categorías sociales que presumen internamente homogéneas. En general, tales ocasiones pueden reducirse a tres tipos: aquellas en las que se utiliza el concepto de Tonnies de comunidad u otro similar, dando a entender que en estos sistemas sociales la integración es sumamente eleva$\mathrm{da}$; aquellas otras en las que se utilizan categorías colectivas típicas de Durkheim, entre las que encuadro el concepto de clase social desarrollado por la sociología marxista; y también al individualismo metodológico, ya que parte de la idea de homogeneidad humana, de que todos los hombres son igualmente racionales e intercambiables. De acuerdo con la hipótesis aquí expuesta, ninguna de estas tres totalidades teóricas es formalmente defendible. Otra cosa es que sean útiles para explicar parcelas de la realidad social, pese al error de punto de partida o error en el axioma suyo inicial.

De todas ellas, las que más deberían reciclarse de acuerdo con lo aquí expuesto son las pertenecientes al segundo tipo, es decir, las categorías colectivas durkheimianas, objetivadas y reificadas generalmente. Entre ellas 
destaco, en particular, el concepto de clase social $^{13}$. Como ejemplo de error de punto de partida en el uso del concepto de clase social, vale la pena recordar el libro de Paul Willis Aprendiendo a trabajar, publicado en castellano ${ }^{14}$ no hace mucho, y que ha gozado de particular interés por parte del público, a juzgar por el rápido agotamiento de la edición. La hipótesis del trabajo es la típica de la sociología marxista de la educación: la subcultura de clase es el mecanismo del que se vale la sociedad para reproducirse a sí misma. Más concretamente, la subcultura de clase obrera impide a los jóvenes que pertenecen a ella aceptar la disciplina de la escuela, con lo que, inevitablemente, quedan ya automarginados de la carrera educativa y, de paso, de la posible movilidad social mediante la educación, por lo que se verán abocados a continuar en la clase obrera de origen. El trabajo de campo de P. Willis está dirigido a desmenuzar los elementos constitutivos de tal subcultura obrera.

El error del autor radica en que confunde la subcultura de clase obrera con una de las opciones posibles en la definición del papel de alumno en la escuela estudiada: la de los «iguales». Fijémonos que en la escuela susodicha existen alumnos de ambos tipos; en otras palabras, que se producen las dos formas antedichas de definición del rol de alumno: desde el punto de vista de los «iguales» («los colegas», de acuerdo con la acertada terminología del traductor al castellano del texto citado de P. Willis) y desde el punto de vista del profesor ( $\ll$ los pringaos»). Pero dejemos al propio autor:

«La dimensión más explícita, más evidente y básica de la cultura contraescolar, es la de su acérrima oposición, en los planos personal y general, a la "autoridad". Este sentimiento es fácilmente verbalizado por los "colegas", el título escogido por aquellos que participan de la cultura contraescolar... (p. 23).

Esta oposición implica una inversión aparente de los valores normales mantenidos por el principio de autoridad. La diligencia, la deferencia, el respeto, empiezan a tener lecturas muy distintas...

${ }^{13}$ El concepto en cuestión ha sufrido un notable abandono por parte de la sociología en general, debido, seguramente, a la «rigidez» propia del mismo, rigidez que ha ido apareciendo en sus múltiples y sucesivas conceptualizaciones. Un intento de matizar al máximo el concepto en cuestión, pero manteniendo la inflexibilidad que le caracteriza, se encuentra en P. Bourdiev, La distinction. Critique sociale du jugement, París, Minuit, 1979 (hay traducción castellana en Tecnos), pp. 112 y ss.

${ }^{14}$ P. Willis, Aprendiendo a trabajar, Madrid, Akal, 1988. El trabajo de campo en el que se basa el texto se llevó a cabo entre 1972 y 1975. Es un interesante libro que pone al descubierto aspectos del sistema educativo ignorados por aquellos estudiosos que sólo se preocupan de los grandes números. Lleva a cabo el autor una descripción cualitativa de lo que ocurre entre los alumnos de una escuela de barrio obrero inglesa, si bien los resultados podrían ser generalizables a cualquier escuela de barrio obrero de Occidente. 
Esta oposición se expresa, principalmente, como un estilo que se vive de infinitas formas, específicas de la institución escolar, que los profesores reconocen instantáneamente y que constituye un aspecto casi ritual de la estructura vital cotidiana de los chicos. Los profesores son expertísimos teóricos de la conspiración. No tiene más remedio... (p. 24).

Los "colegas" son especialistas en un resentimiento reprimido que se detiene siempre justo antes de la confrontación abierta. Metidos en clase, lo más agrupados que pueden, hacen un ruido continuo arrastrando las sillas, abucheando a la menor sugerencia, y no paran de moverse, cambiando de sitio o cambiándose en los asientos. Durante las horas de estudio, algunos muestran abiertamente su desprecio...» (p. 25).

Junto a éstos, los «colegas», existen otros alumnos muy diferentes desde el punto de vista escolar pero que pertenecen al mismo barrio, es decir, pertenecen a la clase obrera también. Se trata de los «pringaos»:

«... los conformistas de la escuela, los "pringaos" para los chavales, tienen una orientación distinta. No se trata tanto de que aguanten a los profesores como a "la idea" de profesor...

Esta apariencia de entusiasmo y complicidad con la autoridad inmediata es lo que convierte a los alumnos conformistas de la escuela en "pringaos" u "orejas"... (p. 26).

Su respuesta a los "colegas" consiste, principalmente, en sufrir miedos ocasionales, incómodos celos y ansiedad general por no caer en la misma red disciplinaria y un sentimiento de frustración porque los "colegas" puedan impedir el normal desarrollo del proceso educativo. Lo que han invertido en el sistema formal y el sacrificio que hacen mientras los otros se están divirtiendo (tanto como el grado de temor que están padeciendo), hace que los alumnos conformistas esperen de los líderes reconocidos del sistema, el personal docente, que se enfrenten a la transgresión, más que impulsarles a suprimirla ellos mismos» (p. 29).

La atención del autor se dirige fundamentalmente hacia los «colegas», especimen que tipifica la subcultura de clase obrera, según él; mientras que el «pringao» parece pertenecer a otro género social. La lectura del texto nos recuerda por completo la idea de T. Parsons anteriormente expuesta; lástima que el autor no tuviera noticia de ella, ya que se trata de la verificación más precisa que se pudiera hacer de la hipótesis en cuestión. Se trata de una versión incluso mejorada, ya que es difícil imaginar a T. Parsons hablando de «pringaos» y de «colegas».

Verdaderamente, el tema a explicar en el trabajo de Willis hubiera debido ser otro. En primer lugar, debería haber buscado una explicación, 
en el entorno en que se movía, al tamaño de las respectivas proporciones de uno y otro tipo de alumnos, es decir, debería haber buscado las causas de que se diera más abundantemente un tipo de definición que otro. Asimismo, debería haber explicado por qué se había llegado a tal situación de radicalización en el conflicto de definiciones en el rol de alumno. Seguramente, tales explicaciones nos hubieran llevado de nuevo al concepto de clase social, pero en este caso hubiera dejado de tener el concepto el sesgo de inevitabilidad y objetividad que le acompaña.

La hipótesis defendida hasta aquí, basada en el concepto de relaciones de roles, en el caso de ser convenientemente revalidada, podría llegar a explicar al menos el origen de una gran cantidad de comportamientos sociales muy heterogéneos. En primer lugar, se encuentra en la base del éxito o del fracaso escolar, tal y como lo explicó T. Parsons; así como en las diferentes conductas en casa de cada uno de los cónyuges, de acuerdo con E. Bott. Por extensión, se puede pensar que tiene que ver con el origen de la mayoría de los procesos que supongan algún tipo de quiebra en el comportamiento de las colectividades.

Este es el caso de la marginalidad; de los comportamientos desviados; de la delincuencia, en especial en la etapa de juventud. Asimismo, cabe la posibilidad de explicar mediante las relaciones de roles otros tipos de rompimiento menos drástico, tal y como las posturas feministas o, en su caso, machistas, entre los propios hombres o entre las propias mujeres. Encajan en esto, también, muchos tipos de rechazo a la jerarquía, tanto formal como informal; las posturas de oposición a la autoridad establecida; los movimientos críticos al orden comúnmente aceptado.

De la misma forma puede ser explicado el origen de las escisiones teóricas basadas, aparentemente, en paradigmas opuestos; las heterodoxias y los cismas supuestamente heréticos. Por último, en esta relación, es posible que el incremento de la complejidad de la división del trabajo tenga que ver algo con las relaciones de roles y, particularmente, con muchos aspectos de las motivaciones a la movilidad social por parte de los actores que la sufren. Entre otras posibilidades. 